\title{
On Grothendieck's Problem of Topologies
}

by

\author{
Julio ALCÁNTARA-BODE****
}

\begin{abstract}
Some classes of locally convex spaces $E$ and $F$ are identified for which not every bounded set of the completed projective tensor product, $E \hat{\otimes} F$, is contained in the bipolar of some set $A \otimes B$, where $A$ is bounded in $E$ and $B$ is bounded in $F$.
\end{abstract}

\section{Statement and Proof of Results}

One of the open problems in the theory of Topological Tensor Products is to characterize classes of locally convex spaces (l.c.s.) $E$ and $F$, for which every bounded subset of the completed projective tensor product, $E \hat{\otimes} F$, is contained in the bipolar of some set $A \otimes B$, where $A$ and $B$ are bounded sets in $E$ and $F$ respectively (Problem of Topologies). For instance it is not known if the Problem of Topologies has a positive answer when $E$ and $F$ are general Fréchet spaces. But if one of the Fréchet spaces is nuclear, then it does ([4], Theorem 21.5.8).

It is the purpose of this note to investigate the Problem of Topologies for classes of l.c.s. that arise in the Theory of Distributions.

Before stating our main result, we make some remarks about the notation. In all that follows $\phi \equiv \bigoplus_{i=1}^{\infty} C$ will be equipped with its largest locally convex topology and $\omega \equiv \prod_{i=1}^{\infty} \boldsymbol{C}$ with its product topology. An $L F$-space will be a strict inductive limit of a sequence of Fréchet spaces.

Communicated by S. Matsuura, October 26, 1984.

Institut für Theoretische Physik, Universität Göttingen, Federal Republic of Germany.

* Alexander von Humboldt Research Fellow (1983-84).

** On leave from the U.P.C.H. Lima-Perú. 
Theorem. The Problem of Topologies for $E \hat{\otimes} F$ has a negative solution if, either

(i) $E$ and $F$ are strong duals of LF-spaces, where one of these LFspaces contains a non-normable Fréchet subspace, the other one is not a Fréchet space and either of them is nuclear, or

(ii) $E$ is a Fréchet space that does not have a continuous norm and $F$ a l.c.s. that contains $\phi$ as a complemented subspace.

Three Lemmas will precede the proof of the Theorem.

Lemma 1. Let $E$ be a l.c.s. that has a non-normable Fréchet subspace and $F$ a l.c.s. containing $\phi$ as a complemented subspace. Then $E \hat{\otimes} F$ and the completed inductive tensor product, $E \bar{\otimes} F$, have different duals.

Proof. First let's assume that $E$ is a non-normable Fréchet space. As $E$ is non-normable and metrizable, there exists a family of continuous linear functionals $\left\{\phi_{k}\right\}_{k \geqq 1}$ on $E$, such that for no sequence of strictly positive numbers $\left\{\alpha_{k}\right\}_{k \geqq 1}$, is $\left\{\alpha_{k} \phi_{k}\right\}_{k \geqq 1}$ equicontinuous ([1], Proposition 1.7. (b)). Let $\phi_{k}$ be a continuous linear functional on $\phi$ whose kernel is $\bigoplus_{n=1}^{\infty} D_{n}^{(k)}$, where $D_{k}^{(k)}=\{0\}$ and $D_{n}^{(k)}=\boldsymbol{C}$ if $n \neq k$. Then $\sum_{k=1}^{\infty} \phi_{k} \psi_{k}$ is a separately continuous bilinear form on $E \times \phi$ which is not jointly continuous. As all tensor product topologies respect complemented subspaces, we get that $(E \bar{\otimes} F)^{\prime} \neq(E \widehat{\otimes} F)^{\prime}$.

A Hahn-Banach extension argument applied to the sequence $\left\{\phi_{k}\right\}_{k \geqq 1}$ gives the proof in the general case.

Lemma 2. Every LF-space that is not a Fréchet space, has $\phi$ as a complemented subspace.

Proof. Let $\left\{E_{j}\right\}$ be a defining sequence of Fréchet subspaces for $E$. There is a sequence $\left\{x_{j}\right\}_{j \geqq 1}$ in $E$ such that $x_{j} \in E_{j}$ and $x_{j+1} \notin E_{j}$ for all $j \geqq 1$. If $F_{j}$ is the smallest subspace containing $\left\{x_{i}\right\}_{1 \leqq i \leqq j}$, an inductive argument shows that there are topological complements $G_{j}$ for $F_{j}$ in $E_{j}$ such that $G_{j+1} \supset G_{j}$ for all $j \geqq 1$. By construction $G=\bigcup_{j=1}^{\infty} G_{j}$ is a subspace of countable codimension of the barrelled space $E$ and 
therefore by the Saxon-Levin-Valdivia Theorem, $G$ is barrelled([6], Theorem 15-1-10). Since $G$ is barrelled and is a union of a strictly increasing sequence of closed subspaces, it has the strict inductive limit topology ([6], Definition 13-3-14 and Theorem 13-3-15). So $G$ is complete and therefore closed. As $E$ is barrelled and $G$ a closed subspace of countable codimension, $F=\bigcup_{j=1}^{r} F_{j}$, with its largest locally convex topology, is a complement of $G$ ([6], Theorem 13-3-14). But $F$ with its largest convex topology is isomorphic to $\phi$ (cf. [6], Problem 12-1-5).

Lemma 3. Let $E$ and $F$ be LF-spaces, one of them nuclear. Then $E \bar{\otimes} F$ is an $L F$-space and its strong dual is isomorphic to $E_{\beta}^{\prime} \hat{\otimes} F_{\beta}^{\prime}$.

Proof. The first assertion follows from the fact that the inductive tensor product topology respects inductive limits ([5], p.96 and p. 119, Exercise 22). Nuclearity guarantees that the inductive limit is strict (cf. [1], Theorem 1.4).

As $E \hat{\otimes} F$ and $E \bar{\otimes} F$ induce the same topology on the Fréchet subspaces of $E \bar{\otimes} F$, and every bounded subset of $E \bar{\otimes} F$ is contained in one of these subspaces, it follows from the Hahn-Banach theorem that $(E \hat{\otimes} F)^{\prime}$ is strongly dense in $(E \bar{\otimes} F)^{\prime}$. By the nuclear theorem and the positive solution of the problem of Topologies for $E \bar{\otimes} F$ ([4], Theorem 21.5.8), $E^{\prime} \otimes F^{\prime}$ is strongly dense in $(E \hat{\otimes} F)^{\prime}$ and therefore also in $(E \bar{\otimes} F)^{\prime}$. Since the strong dual of a nuclear $L F$-space is nuclear ([4], Proposition 21.5.1 and Corollary 21.5.5) and $(E \bar{\otimes} F)_{\beta}^{\prime}$ is complete $(E \bar{\otimes} F$ is bornological), it will be enough to show that the strong topology on $(E \bar{\otimes} F)^{\prime}$ induces the injective topology $\varepsilon$ on its dense subspace $E^{\prime} \otimes F^{\prime}$. When $A$ and $B$ vary over all possible absolutely convex closed bounded sets in $E$ and $F$, respectively, the seminorms

$$
P_{A, B}(\xi)=\sup _{z \in\langle\overline{A \otimes} \bar{B}>}|\xi(z)|, \quad \xi \in(E \bar{\otimes} F)^{\prime},
$$

$(\langle\overline{A \otimes B}\rangle \equiv$ absolutely closed convex hull of $A \otimes B$ ) define the strong topology on $(E \vec{\otimes} F)^{\prime}$. But

$$
P_{A, B}\left(\sum_{l=1}^{k} \phi_{l} \otimes \phi_{l}\right)=\sup _{x \in A, Y \in B}\left|\sum_{l=1}^{k} \phi_{l}(x) \phi_{l}(y)\right|
$$




$$
=\left(P_{A} \bigotimes_{\varepsilon} q_{B}\right)\left(\sum_{l=1}^{k} \phi_{l} \otimes \phi_{l}\right), \phi_{l} \in E^{\prime}, \phi_{l} \in F^{\prime} \quad(1 \leqq l \leqq k)
$$

where $P_{A}(\phi) \equiv \sup _{x \in A}|\phi(x)|$ and $q_{B}(\phi) \equiv \sup _{y \in B}|\phi(y)|$.

This finishes the proof.

\section{Proof of Theorem.}

(i) By Lemmas 1 and 2 there is a continuous seminorm $p$ on $E \bar{\otimes} F$ whose restriction to $E \otimes_{\pi} F$ ( $E \otimes F$ equipped with the projective topology) is not continuous. The polar $B_{p}^{0}$ of the unit ball $B_{p}$ of this seminorm is a bounded set in $(E \bar{\otimes} F)_{\beta}^{\prime} \cong E_{\beta}^{\prime} \hat{\otimes} F_{\beta}^{\prime}$, such that for no absolutely convex closed bounded sets $A$ and $B$ in $E_{\beta}^{\prime}$ and $F_{\beta}^{\prime}$, respectively, $B_{p}^{0} \subset\langle\overline{A \otimes B}\rangle$. In effect this inclusion and the barrelledness of $E$ and $F$ would imply, as in the proof of Lemma 3, that the restriction of $p$ to $E \otimes_{\pi} F$ would be continuous.

(ii) By Lemma 3 if $E=\phi$ and $F=\omega$ we have $(\phi \bar{\otimes} \omega)_{\beta}^{\prime} \cong \omega \hat{\otimes} \phi$ as $\phi_{\beta}^{\prime} \cong \omega$ and $\omega_{\beta}^{\prime} \cong \phi$. By part (i), this proves part (ii) when $E=\omega$. The rest of the proof follows from the fact that every Fréchet space without a continuous norm has $\omega$ as a complemented subspace ([4], Theorem 7.2.7).

Remark (a). By Lemma 2, part (ii) of the Theorem applies to $E_{\beta}^{\prime} \hat{\otimes} F$ if $E$ and $F$ are LF-spaces that are not Fréchet.

Remark (b). It can be shown that if $E$ and $F$ are arbitrary locally convex direct sums of Fréchet spaces equipped with continuous norms, then as sets $E \hat{\otimes} F=E \bar{\otimes} F$ and both completions have the same bounded sets. In particular this applies when $E$ and $F$ are $L F$-spaces with continuous norms and unconditional bases ([2], Lemma 5.43 and Exercise 5.59). Therefore a positive solution of the Problem of Topologies under these conditions for $E \bar{\otimes} F$ implies a positive solution of the corresponding problem for $E \hat{\otimes} F$. This applies, for example, when $E$ and $F$ are $L F$-spaces with unconditional bases, continuous norms, and one of them is nuclear.

Remark (c). A positive solution of the Problem of Topologies is 
useful for deriving some general factorization Theorems in Quantum Field Theory (cf. [3], Proposition 3.1).

\section{Acknowledgments}

Part of this work was presented at the Göttingen Symposium on Algebraic Quantum Field Theory and Rings of Unbounded Operators (6. -11. September 1982) and represents research carried out at the Open University (England) and at the Universität Göttingen. I should like to thank these institutions and the Alexander von HumboldtStiftung for financial support, the U.P.C.H. (Lima-Perú) for an extended leave of absence and Professor H.J. Borchers for the hospitality extended to me at Göttingen. I am also grateful to Dr. K.-D。 Kürsten for pointing out an inaccuracy in an earlier proof of Lemma 3.

\section{References}

[1] J. Alcántara and D. A. Dubin, I*-Algebras and their Applications, Publ. R.I.M.S., Kyoto University, $\mathbb{1 7}$ (1981), 179-199.

[2] S. Dineen, Complex Analysis in Locally Convex Spaces, North-Holland, Amsterdam, 1981.

[ 3 ] G. C. Hegerfeldt, Prime Field Decompositions and Infinitely Divisible States on Borchers' Tensor Algebra, Commun. Math. Phys., 45 (1975), 137-151.

[4] H. Jarchow, Locally Convex Spaces, Teubner, Stuttgart, 1981.

[5] H.H. Schaefer, Topological Vector Spaces, Springer, Berlin, 1971.

[6] A. Wilansky, Modern Methods in Topological Vector Spaces, McGraw-Hill, New York, 1978. 
